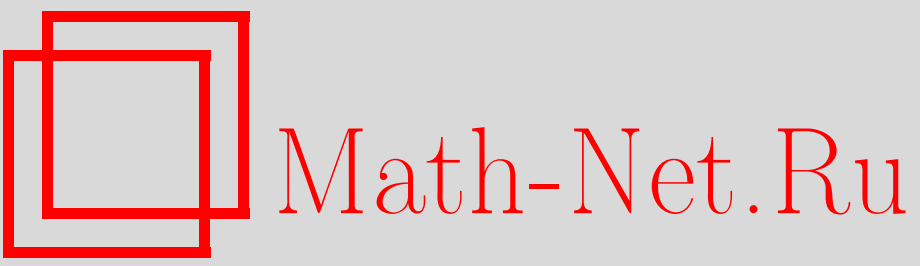

M. Yamazato, Absolute continuity conditions for multivariate infinitely divisible distributions and their applications, Теория вероятн. и ее примен., 2011, том 56, выпуск 2, 351-366

DOI: https://doi.org/10.4213/tvp4378

Использование Общероссийского математического портала Math-Net.Ru подразумевает, что вы прочитали и согласны с пользовательским соглашением

http://www. mathnet.ru/rus/agreement

Параметры загрузки:

IP: 3.93 .64 .190

26 апреля 2023 г., 17:52:44 
2011

(c) 2011 г.

YAMAZATO M.*

\title{
ABSOLUTE CONTINUITY CONDITIONS FOR MULTIVARIATE INFINITELY DIVISIBLE DISTRIBUTIONS AND THEIR APPLICATIONS ${ }^{1)}$
}

\begin{abstract}
Приводятся два достаточных условия абсолютной непрерывности относительно меры Лебега многомерных безгранично делимых распределений. Эти условия применяются для доказательства абсолютной непрерывности безгранично делимых распределений, меры Леви которых имеют обобщенное полярное разложение с абсолютно непрерывной радиальной частью указанного вида. Более того, одно из этих условий применяется для доказательства необходимого и достаточного условия абсолютной непрерывности переходных вероятностей процессов типа Орнштейна-Уленбека, управляемых процессами Леви.
\end{abstract}

Ключевые слова и фразы: абсолютная непрерывность, многомерные безгранично делимые распределения, процессы типа Орнштейна-Уленбека.

1. Introduction and main results. Let $\mu$ be an infinitely divisible distribution on $\mathbf{R}^{d}$ with characteristic function

$$
\widehat{\mu}(\theta)=\int e^{i\langle\theta, x\rangle} \mu(d x)=\exp \left\{\int_{\mathbf{R}^{d}}\left(e^{i\langle\theta, x\rangle}-1-i\langle x, \theta\rangle 1_{\{|x| \leqslant 1\}}(x)\right) \nu(d x)\right\},
$$

where $\theta \in \mathbf{R}^{d}$ and $\nu$ is a measure on $\mathbf{R}^{d}$ with $\int\left(|x|^{2} \wedge 1\right) \nu(d x)<\infty$ and $\nu(\{0\})=0$. The measure $\nu$ is called the Lévy measure of $\mu$. For a Lévy measure $\nu$, define a finite measure $\widetilde{\nu}$ by $\widetilde{\nu}(d x)=|x|^{2} /\left(1+|x|^{2}\right) \nu(d x)$. We denote by $\rho^{r *}$ the $r$-fold convolution of a finite measure $\rho$.

In this paper, we are interested in under what condition, $\mu$ is absolutely continuous with respect to the Lebesgue measure on $\mathbf{R}^{d}$. In the above representation, there is no Gaussian part. In order to consider the absolute continuity problem, the case with Gaussian part is not so interesting. So, we omit this case. It is known [3] that if $\nu\left(\mathbf{R}^{d}\right)=\infty$ and $\widetilde{\nu}^{r *}$ is absolutely continuous with respect to the Lebesgue measure on $\mathbf{R}^{d}$ for some positive integer $r$, then $\mu$ is absolutely continuous. If $\nu$ satisfies GPDACR property

*Department of Mathematical Sciences, University of the Ryukyus, Senbaru 1, Nishihara-cho, Okinawa, 903-0213, Japan; e-mail: yamazato@math.u-ryukyu.ac.jp

1) Supported partially by JSPS KAKENHI 22540145. 
stated in Section 4 and there is no $(d-2)$-dimensional linear subspace having positive or infinite mass and $\nu\left(H^{c}\right)=\infty$ for every $(d-1)$-dimensional linear subspace $H$, then $\widetilde{\nu}^{d *}$ is absolutely continuous with respect to the Lebesgue measure on $\mathbf{R}^{d}$. Also, in case that there are finitely many proper linear subspaces on which the Lévy measure $\nu$ has positive or infinite masses, we can easily conclude whether $\mu$ is absolutely continuous or not. However, if there are infinitely many $(d-1)$-dimensional linear subspaces on which $\nu$ has finite positive masses, then the situation become very complicated. So many cases should be considered. Therefore, we concentrate ourselves on such cases.

For a linear subspace $W$ of $\mathbf{R}^{d}$, denote by $\Lambda_{W}$ the Lebesgue measure on $W$. For two measures $\mu_{1}$ and $\mu_{2}$, we use the notation $\mu_{1} \ll \mu_{2}$ if $\mu_{1}$ is absolutely continuous with respect to $\mu_{2}$.

Theorem 1.1. Let $d \geqslant 1$. Assume that there is an integer $r \geqslant 1$ such that

1) $\nu$ is represented as $\nu=\sum_{k=1}^{\infty} \nu_{k}$, where each $\nu_{k}$ is concentrated on a linear subspace $H_{k}$ and it has no mass on any proper linear subspace of $H_{k}$ and $\left(\widetilde{\nu}_{k}\right)^{r *} \ll \Lambda_{H_{k}}$

2) $\sum_{k}\left(\nu_{k}\left(K^{c}\right)\right)^{r}=\infty$ for any $(d-1)$-dimensional linear subspace $K$ of $\mathbf{R}^{d}$.

Then $\mu \ll \Lambda_{\mathbf{R}^{d}}$.

Let $\nu^{\ell}$ be a measure defined by removing masses of $\nu$ on all $(\ell-1)$ dimensional linear subspaces. We denote by $\left\{H_{k}^{(\ell)}\right\}$ the totality of $\ell$ dimensional linear subspaces satisfying $0<\nu^{\ell}\left(H_{k}^{(\ell)}\right) \leqslant \infty$ and define $\nu^{(\ell)}=\nu^{\ell} \mid \bigcup_{k} H_{k}^{(\ell)}$.

$\mathrm{R}$ e $\mathrm{m}$ a $\mathrm{rk}$ 1.1. 1) In the assumptions in Theorem 1.1, $H_{k}$ are not restricted to proper linear subspaces and not assumed to be distinct. We rearrange $\left\{H_{k}\right\}$ as $\left\{H_{\ell, k}\right\}$, where $\ell$ is the dimension of $H_{\ell, k}$ for each $\ell \leqslant 1$, and define $\nu_{\ell, k}=\nu_{j}$ for $H_{j}=H_{\ell, k}$. Then $\nu^{(\ell)}=\sum_{k} \nu_{\ell, k}$.

2) Assumption 1 in Theorem 1.1 is weaker than the assumption that $\left(\left.\widetilde{\nu}^{(\ell)}\right|_{H_{k}^{(\ell)}}\right)^{r *} \ll \Lambda_{H_{k}^{(\ell)}}$ since for $H_{k}^{(\ell)}=H_{k_{1}}=H_{k_{2}}=\cdots,\left(\left.\widetilde{\nu}^{(\ell)}\right|_{H_{k}^{(\ell)}}\right)^{r *} \ll \Lambda_{H_{k}^{(\ell)}}$ implies that $\widetilde{\nu}_{k_{1}}^{r *}, \widetilde{\nu}_{k_{2}}^{r *}, \ldots \ll \Lambda_{H_{k}^{(\ell)}}$ but not vice versa. Note that Assumption 1 is weaker than $\widetilde{\nu}^{r *} \ll \Lambda_{\mathbf{R}^{d}}$.

3) Tucker [7] shows that if $\nu \ll \Lambda_{\mathbf{R}^{d}}$ and $\nu\left(\mathbf{R}^{d}\right)=\infty$, then $\mu \ll \Lambda_{\mathbf{R}^{d}}$. This is equivalent to Theorem 4.1 in $d=1$ and the assumption of Theorem 1.1 is weaker by the above argument even in one-dimension.

Corollary 1.1. Assume that for each $1 \leqslant k \leqslant d,\left.\widetilde{\nu}^{(k)}\right|_{H} \ll \Lambda_{H}$ on each $k$-dimensional linear subspace $H$ with $0<\nu^{(k)}(H) \leqslant \infty$. Then $\mu \ll \Lambda_{\mathbf{R}^{d}}$ if and only if $\nu\left(K^{c}\right)=\infty$ for any $(d-1)$-dimensional linear subspace $K$.

This corollary is straightforward from Theorem 1.1 setting $r=1$.

Now, we describe another type result. For a linear subspace $W$ of $\mathbf{R}^{d}$, denote by $T_{W}$ the orthogonal projection operator onto $W$. Denote $\prod_{i=1}^{r} H_{i}=$ 
$H_{1} \times \cdots \times H_{r}$ and let

$K\left(H_{1}, \ldots, H_{r}\right)=\left\{\left(x_{1}, \ldots, x_{r}\right) \in \prod_{i=1}^{r} H_{i}: x_{1}, \ldots, x_{r}\right.$ are linearly independent $\}$ for linear subspaces $H_{1}, \ldots, H_{r}$.

Theorem 1.2. Let $d \geqslant 1,1 \leqslant \ell \leqslant d$ and let $W$ be an $m$-dimensional linear subspace of $\mathbf{R}^{d}$ with $\nu^{(\ell)}(W)>0$ for $\ell \leqslant m \leqslant d$ or $W=\{0\}$. Let $T:=T_{W^{\perp}}$ and $T \nu_{\ell}(\cdot):=\nu^{(\ell)}\left(T^{-1}(\cdot) \cap W^{c}\right)$. Let $\left\{V_{k}\right\}$ be a sequence of all $j$-dimensional linear subspaces of $W^{\perp}$ such that $0<T \nu_{\ell}\left(V_{k}\right) \leqslant \infty$. Regard $m=0$ and $j=\ell$ if $W=\{0\}$. Suppose that if $\operatorname{dim}\left(V:=\sum_{i=1}^{r} V_{k_{i}}\right)=r$ $(1 \leqslant j \leqslant r \leqslant d-m)$, then

$$
\int_{K\left(V_{k_{1}}, \ldots, V_{k_{r}}\right)} 1_{Z}\left(\sum_{i=1}^{r} x_{k_{i}}\right) \prod_{i=1}^{r}\left(T \widetilde{\nu}_{\ell}\right)^{(j)}\left(d x_{k_{i}}\right)=0
$$

for any Borel subset $Z$ of $V$ with $\Lambda_{V}(Z)=0$. Then $\mu \ll \Lambda_{\mathbf{R}^{d}}$ if and only if

$$
\nu\left(K^{c}\right)=\infty
$$

for any $(d-1)$-dimensional linear subspace $K$ of $\mathbf{R}^{d}$.

R e m a r k 1.2. 1) Regard $H_{1}^{(\ell)}=H_{2}^{(\ell)}=\cdots=\mathbf{R}^{d}$ if $\ell=d$ in the above theorem. Hence, if $d=1$, then condition (2) means that $\nu \ll \Lambda_{\mathbf{R}^{d}}$.

2) The results in Sections 4 and 5 show that the assumption in the above theorem is not empty and useful.

In the following section, we prepare lemmas necessary for the proofs of the above theorems. In Section 3, we give the proofs of Theorems 1.1 and 1.2. In Section 4, we apply the main results to absolute continuity of multivariate infinitely divisible distributions under GPDACR condition for the Lévy measure, which is defined in Section 4. Section 5 is devoted to a proof of the absolute continuity of transition probabilities of the socalled processes of Ornstein-Uhlenbeck type (OU type processes) in [5] as an application of Theorem 1.2.

\section{Lemmas.}

Lemma 2.1. Let $H_{1}$ and $H_{2}$ be linear subspaces of $\mathbf{R}^{d}$ such that $\operatorname{dim}\left(H_{1}+H_{2}\right)=d$. If $\mu_{1} \ll \Lambda_{H_{1}}$ and $\mu_{2} \ll \Lambda_{H_{2}}$, then $\mu_{1} * \mu_{2} \ll \Lambda_{\mathbf{R}^{d}}$.

Lemma 2.2. Let $H \neq\{0\}$ be a proper linear subspace of $\mathbf{R}^{d}$ and $H^{\perp}$ its orthogonal complement in $\mathbf{R}^{d}$. Let $T=T_{H^{\perp}}$. Let $\mu_{1}(d x)=q(d x)+p(x) d x$ be a probability measure on $H$ and $\mu_{2}$ a probability measure on $\mathbf{R}^{d}$ such that $T \mu_{2} \ll \Lambda_{H^{\perp}}$, where $T \mu_{2}(\cdot)=\mu_{2}\left(T^{-1}(\cdot)\right)$. Then $\mu(Z)=\mu_{1} * \mu_{2}(Z) \leqslant q:=$ $q(H)$ for any Borel set $Z$ in $\mathbf{R}^{d}$ with $\Lambda_{\mathbf{R}^{d}}(Z)=0$. 
Pr o o f. For $x \in \mathbf{R}^{d}$, we denote $x_{2}=T x$ and $x_{1}=x-x_{2}$. Let $Y$ be a random variable with distribution $\mu_{2}$. Let $P_{1}\left(d y_{1} \mid y_{2}\right)=\mathbf{P}(Y-T Y \in$ $\left.d y_{1} \mid T Y=y_{2}\right)$ be the conditional probability of $Y-T Y$ given $T Y=y_{2}$. Since

$$
\int_{H^{\perp}} d y_{2} \int_{H} 1_{Z}\left(x_{1}, y_{2}\right) d x_{1}=0
$$

there is a Borel set $Z_{2}$ in $H^{\perp}$ with $\Lambda_{H^{\perp}}\left(Z_{2}\right)=0$ such that for every $y_{2} \notin Z_{2}$

$$
\int_{H} 1_{Z}\left(x_{1}, y_{2}\right) d x_{1}=0
$$

Let

$$
\begin{aligned}
h_{1}\left(y_{1}, y_{2}\right) & =\int_{H} 1_{Z}\left(x_{1}+y_{1}, y_{2}\right) p\left(x_{1}\right) d x_{1}, \\
h\left(y_{1}, y_{2}\right) & =h_{1}\left(y_{1}, y_{2}\right)+\int_{H} 1_{Z}\left(x_{1}+y_{1}, y_{2}\right) q\left(d x_{1}\right),
\end{aligned}
$$

which are Borel measurable in $\left(y_{1}, y_{2}\right)$, where $y_{1} \in H$ and $y_{2} \in H^{\perp}$. Then we have

$$
h_{1}\left(y_{1}, y_{2}\right)=1_{Z_{2}}\left(y_{2}\right) h_{1}\left(y_{1}, y_{2}\right)
$$

and

$\mu(Z)=\int_{\mathbf{R}^{d}} \mu_{2}(d y) h\left(y_{1}, y_{2}\right) \leqslant \int_{Z_{2}} T \mu_{2}\left(d y_{2}\right) \int_{H} h_{1}\left(y_{1}, y_{2}\right) P_{1}\left(d y_{1} \mid y_{2}\right)+q=q$.

Lemma 2.2 is proved.

The above lemma is an extension of a result in the proof of Theorem 27.10 in [4]. The proof in [4] treats the case $q=0$. In other words, absolute continuity of $\mu_{1}$ is assumed.

Lemma 2.3. Let $\left\{a_{j}\right\}$ and $\left\{b_{j}\right\}$ be sequences of positive numbers. Assume that $r \geqslant 1$. Then $\sum_{j}\left(a_{j}+b_{j}\right)^{r}<\infty$ if and only if $\sum_{j}\left(a_{j}\right)^{r}<\infty$ and $\sum_{j}\left(b_{j}\right)^{r}<\infty$.

P r o o f. By Minkowski's inequality

$$
\sum_{j}\left(a_{j}+b_{j}\right)^{r} \leqslant\left(\left(\sum_{j}\left(a_{j}\right)^{r}\right)^{1 / r}+\left(\sum_{j}\left(b_{j}\right)^{r}\right)^{1 / r}\right)^{r}
$$

and the inequality $a^{r}+b^{r}<(a+b)^{r}$ for $a, b>0$, we have the conclusion.

Lemma 2.4. Let $W$ be a linear subspace of $\mathbf{R}^{d}$ and let $T=T_{W^{\perp}}$. Let $H_{1}, H_{2}, \ldots$ be distinct $r$-dimensional linear subspaces of $\mathbf{R}^{d}$ such that $H_{k} \nsubseteq$ $W$ and $W \nsubseteq H_{k}$ for $k \geqslant 1$. Assume that for each $k \leqslant 1,0<\nu^{(r)}\left(H_{k}\right)<\infty$ and $\left(\left.\nu^{(r)}\right|_{H_{k}}\right)^{r *} \ll \Lambda_{H_{k}}$. Then $\left(T\left(\left.\nu^{(r)}\right|_{H_{k}}\right)\right)^{r *} \ll \Lambda_{T\left(H_{k}\right)}$ for each $k$. 
P r o o f. Set $H=H_{k}$. By the assumption, $\left(\left.\nu^{(r)}\right|_{H}\right)^{r *} \ll \Lambda_{H}$. Let $Z$ be a Borel set with $\Lambda_{T(H)}(Z)=0$. We have $\left(\left.T\right|_{H}\right)^{-1}(0)=H \cap W$ and

$$
\left.\operatorname{dim} T\right|_{H}(H)+\operatorname{dim}(H \cap W)=\left.\operatorname{dim} T\right|_{H}(H)+\left(\left.T\right|_{H}\right)^{-1}(0)=r .
$$

We also have that $\operatorname{dim}(H \cap W)+\operatorname{dim}\left(H \cap(H \cap W)^{\perp}\right)=r$. Let $H^{\prime}=$ $H \cap(H \cap W)^{\perp}$. Then $\operatorname{dim} H^{\prime}=\left.\operatorname{dim} T\right|_{H}(H)$. Since $H \cap W$ is a proper linear subspace of $H$ by the assumption, $0 \leqslant \operatorname{dim}(H \cap W)<r$.

We decompose $x \in H$ as $x=x_{1}+x_{2}, x_{1} \in H \cap W$ and $x_{2} \in H^{\prime}$. Then $T x=T x_{2}$. Hence $\left.T\right|_{H^{\prime}}$ is a linear map on $H^{\prime}$ onto $T(H)$. Since $\operatorname{dim} H^{\prime}=\left.\operatorname{dim} T\right|_{H}(H)=\left.\operatorname{dim} T\right|_{H^{\prime}}\left(H^{\prime}\right),\left.T\right|_{H^{\prime}}\left(H^{\prime}\right)$ is a bijection on $H^{\prime}$ onto $T(H)$. Hence $\Lambda_{H^{\prime}}\left(\left.T\right|_{H^{\prime}}\right)^{-1}(Z)=0$.

For $y \in T(H)$, let $x$ be a uniquely determined element of $H^{\prime}$ such that $T x=y$. Assume that for $z \in H \cap W, x+z=w_{1}+w_{2}$ with $w_{1} \in W$ and $w_{2} \in W^{\perp}$. Then, $y=T(x+z)=T\left(w_{1}+w_{2}\right)=w_{2}$ and hence $x+z=w_{1}+y$, i.e., $x+H \cap W \subset(y+W) \cap H$. Conversely, for any $w \in W$ satisfying $y+w \in H$, decompose $y+w$ as $y+w=h_{1}+h_{2}$ with $h_{1} \in H \cap W$ and $h_{2} \in H^{\prime}$. Then, $y=T(y+w)=T\left(h_{1}+h_{2}\right)=T\left(h_{2}\right)$. Thus, $x=h_{2}$ and $(y+W) \cap H \subset$ $x+H \cap W$. Hence, we have $(y+W) \cap H=x+H \cap W$. This means that $T^{-1}(Z) \cap H=\left(\left.T\right|_{H^{\prime}}\right)^{-1}(Z)+H \cap W$. Therefore, $\Lambda_{H}\left(T^{-1}(Z) \cap H\right)=0$ and we have

$$
\left(T\left(\left.\nu^{(r)}\right|_{H}\right)\right)^{r *}(Z)=T\left(\left(\left.\nu^{(r)}\right|_{H}\right)^{r *}\right)(Z)=\left(\nu^{(r)}\right)^{r *}\left(T^{-1}(Z) \cap H\right)=0 .
$$

Lemma 2.4 is proved.

Lemma 2.5. Let $\left\{c_{i}^{(n)}\right\}_{i, n=1,2, \ldots}$ be a sequence of positive numbers such that $\sup _{i, n} c_{i}^{(n)}<\infty$. Let

$$
a_{i}^{(n)}=\sum_{j=1}^{r-1} \frac{\left(c_{i}^{(n)}\right)^{j}}{j !} \quad \text { and } \quad b_{i}^{(n)}=\sum_{j=r}^{\infty} \frac{\left(c_{i}^{(n)}\right)^{j}}{j !}
$$

for some $r \geqslant 1$. If $\lim _{n \rightarrow \infty} \sum_{i=1}^{\infty}\left(c_{i}^{(n)}\right)^{r}=\infty$, then

$$
\lim _{n \rightarrow \infty} \lim _{q \rightarrow \infty} \exp \left(-\sum_{k=1}^{q} c_{k}^{(n)}\right)\left(\prod_{k=1}^{q}\left(1+a_{k}^{(n)}\right)\right)\left(\sum_{1 \leqslant j_{1}<\cdots<j_{p} \leqslant q} \prod_{k=1}^{p} \frac{b_{j_{k}}^{(n)}}{1+a_{j_{k}}^{(n)}}\right)=0
$$

for $p \geqslant 1$.

P r o o f. We have

$$
\begin{gathered}
\frac{\exp \left(\sum_{i=1}^{q} c_{i}^{(n)}\right)}{\prod_{i=1}^{q}\left(1+a_{i}^{(n)}\right)\left(\sum_{1 \leqslant j_{1}<\cdots<j_{p} \leqslant q} \prod_{k=1}^{p} b_{j_{k}}^{(n)} /\left(1+a_{j_{k}}^{(n)}\right)\right)} \\
\geqslant \prod_{i=1}^{q}\left(1+\frac{b_{i}^{(n)}}{1+a_{i}^{(n)}}\right)\left(\sum_{k=1}^{q} \frac{b_{k}^{(n)}}{1+a_{k}^{(n)}}\right)^{-p} .
\end{gathered}
$$


Taking logarithm, we have

$$
\begin{aligned}
& \sum_{i=1}^{q} \ln \left(1+\frac{b_{i}^{(n)}}{1+a_{i}^{(n)}}\right)-p \ln \left(\sum_{k=1}^{q} \frac{b_{k}^{(n)}}{1+a_{k}^{(n)}}\right) \\
& \quad \geqslant \sum_{i=1}^{q} c \frac{b_{i}^{(n)}}{1+a_{i}^{(n)}}-p \ln \left(\sum_{i=1}^{q} \frac{b_{i}^{(n)}}{1+a_{i}^{(n)}}\right) \geqslant c^{\prime} \sum_{i=1}^{q} b_{i}^{(n)}-p \ln \left(\sum_{i=1}^{q} b_{i}^{(n)}\right),
\end{aligned}
$$

where $c$ and $c^{\prime}$ are positive constants and we used $a_{k}^{(n)}, b_{k}^{(n)} \leqslant e^{\sup _{k, n} c_{k}^{(n)}}<\infty$ in the above estimates. Hence the left-hand side of (4) tends to $\infty$ as $q \rightarrow \infty$ and $n \rightarrow \infty$ since

$$
\lim _{n \rightarrow \infty} \sum_{i=1}^{\infty} b_{i}^{(n)} \geqslant \lim _{n \rightarrow \infty} \sum_{i=1}^{\infty} \frac{\left(c_{i}^{(n)}\right)^{r}}{r !}=\infty .
$$

3. Proofs of theorems. In this section, we give proofs of theorems described in Section 1.

Pro of of $\mathrm{Th}$ e or e $\mathrm{m}$ 1.1. We prove the theorem by induction in $d$. Let $d=1$. Let $\nu=\sum_{k} \nu_{k}$, where $\left(\widetilde{\nu}_{k}\right)^{r *} \ll \Lambda_{\mathbf{R}^{1}}$ and $\sum_{k} \nu^{r *}\left(\mathbf{R}^{1}\right)=$ $\infty$. Let $Z$ be a Borel set with $\Lambda_{\mathbf{R}^{1}}=0$. Define $\rho_{k}^{s}=\sum_{i=1}^{r-1}\left(\nu_{k}\right)^{i *} / i$ ! and $\rho_{k}^{a}=\sum_{i=r}^{\infty}\left(\nu_{k}\right)^{i *} / i$ !. Let $a_{k}=\rho_{k}^{s}\left(\mathbf{R}^{1}\right), b_{k}=\rho_{k}^{a}\left(\mathbf{R}^{1}\right)$ and $e^{c_{k}}=1+a_{k}+b_{k}$. If $c_{k}=\infty$ for some $k$, then $\mu \ll \Lambda_{\mathbf{R}^{1}}$. Suppose that $c_{k}<\infty$ for every $k$. Define a probability measure $\mu_{n}$ by

$$
\begin{aligned}
\mu & =e^{-\sum c_{k}}\left(\delta_{0}+\rho_{1}^{s}+\rho_{1}^{a}\right) * \cdots *\left(\delta_{0}+\rho_{n}^{s}+\rho_{n}^{a}\right) * \mu_{n}, \\
\mu(Z) & \leqslant e^{-\sum c_{k}} \prod_{k=1}^{n}\left(1+a_{k}\right)=\left[\prod_{k=1}^{n}\left(1+\frac{b_{k}}{1+a_{k}}\right)\right]^{-1} .
\end{aligned}
$$

If $\sup _{k} c_{k}=\infty$, then we can assume that $c_{k} \uparrow \infty$ as $k \rightarrow \infty$. Then

$$
\left[\prod_{k=1}^{n}\left(1+\frac{b_{k}}{1+a_{k}}\right)\right]^{-1} \leqslant \frac{1+a_{n}}{1+a_{n}+b_{n}} \rightarrow 0
$$

as $n \rightarrow \infty$. If $\sup _{k} c_{k}<\infty$, then we have

$$
\left[\prod_{k=1}^{n}\left(1+\frac{b_{k}}{1+a_{k}}\right)\right]^{-1} \rightarrow 0
$$

as $n \rightarrow \infty$ since $a_{k}, b_{k} \leqslant e^{\sup _{k} c_{k}}$ and $\sum_{k} b_{k}=\infty$. Hence $\mu \ll \Lambda_{\mathbf{R}^{1}}$.

Now, we assume that the theorem is valid for dimension less than $d \geqslant 2$.

Step 1 . We prove the following statement: Let $1 \leqslant \ell \leqslant d-1$ and let $\left\{H_{\ell, k}\right\}$ be a sequence of not necessarily distinct $\ell$-dimensional linear subspaces appearing in assumption 1 as in Remark 1.1. If $\left\{H_{\ell, k}\right\}$ satisfies that $0<\nu_{\ell, k}\left(H_{\ell, k}\right)<\infty$ and $\sum_{k=1}^{\infty} \nu_{\ell, k}\left(H_{\ell, k} \cap K^{c}\right)^{r}=\infty$ for any $(d-1)$ dimensional linear subspace $K$ in $\mathbf{R}^{d}$, then $\mu \ll \Lambda_{\mathbf{R}^{d}}$. 
We denote $\nu_{\ell, k}$ by $\nu_{k}$ and $H_{\ell, k}$ by $H_{k}$. Let $D_{m}=\sup _{W} \sum_{k}\left(\nu_{k}\left(H_{k} \cap W\right)\right)^{r}$, where $W$ is an $m$-dimensional linear subspace of $\mathbf{R}^{d}$. We divide into the following cases: $D_{1} \leqslant \cdots \leqslant D_{m-1}<\infty=D_{m}=\cdots=D_{d}$ for $\ell \leqslant m \leqslant d$.

1. For $\ell \leqslant m \leqslant d-1$, assume that $D_{\ell}, \ldots, D_{m-1}<\infty=D_{m}$. Fix an $m$-dimensional linear subspace $W$ of $\mathbf{R}^{d}$. Let $T=T_{W^{\perp}}$, where $W^{\perp}$ is the orthogonal complement of $W$. Let $\sigma_{1}=\left.\sum_{k} \nu_{k}\right|_{H_{k} \cap W}$ and $\sigma_{2}=\left.\sum_{k} \nu_{k}\right|_{H_{k} \cap W^{c}}$ and let $\mu_{1}$ and $\mu_{2}$ be infinitely divisible distributions determined through (1) by $\sigma_{1}$ and $\sigma_{2}$, respectively. Note that $\operatorname{dim} W^{\perp}=d-m \leqslant d-1$. By the assumption 2 ,

$$
\sum_{k=1}^{\infty}\left(T \nu_{2}\left(H_{k} \cap K^{c}\right)\right)^{r}=\sum_{k}\left(\nu_{k}\left(H_{k} \cap\left(T^{-1}(K)\right)^{c}\right)\right)^{r}=\infty
$$

for any $(d-m-1)$-dimensional linear subspace $K$ of $W^{\perp}$ since $\operatorname{dim} T^{-1}(K)=$ $d-1$. By the assumption 1 and Lemma 2.4, $\left(T\left(\left.\nu\right|_{H_{k}}\right)\right)^{r *} \ll \Lambda_{T\left(H_{k}\right)}$ for each $k$ (it may happen that $T\left(H_{j}\right)=T\left(H_{k}\right)$ for distinct $H_{j}$ and $H_{k}$ ). Hence $T \sigma_{2}$ satisfies the assumption 1 with $W^{\perp}$ as $\mathbf{R}^{d}$. Since $\operatorname{dim} W^{\perp}<d$, infinitely divisible distribution $T \mu_{2}$ corresponding to $T \sigma_{2}$ is absolutely continuous on $W^{\perp}$ by the induction hypothesis.

2. Now, we consider $\mu_{1}$. Rearrange $H_{k}$ in $W$ as $\left\{H_{k}\right\}$ and let $\left\{\nu_{k}\right\}$ be the sequence of corresponding measures. Let $\rho_{k}^{s}=\sum_{n=1}^{r-1} \nu_{k}^{n *} /(n !), \rho_{k}^{a}=$ $\sum_{n=r}^{\infty} \nu_{k}^{n *} /(n !)$, and $\rho_{k}=\delta_{0}+\rho_{k}^{s}+\rho_{k}^{a}$. Let $a_{k}=\rho_{k}^{s}\left(H_{k}\right), b_{k}=\rho_{k}^{a}\left(H_{k}\right)$. Define $\mu_{q}^{1}$ by

$$
\mu_{1}=e^{-\sum_{k=1}^{q}{ }^{c_{k}}} \rho_{1} * \rho_{2} * \cdots * \rho_{q} * \mu_{q}^{1} .
$$

Let $Z$ be a Borel set of $W$ with $\Lambda_{W}(Z)=0$. We have

$$
\begin{aligned}
\rho_{1} * \rho_{2} * \cdots * \rho_{q} * \mu_{q}^{1}(Z)=\left(\prod_{i=1}^{q} *\left(\delta_{0}+\rho_{i}^{s}+\rho_{i}^{a}\right)\right) * \mu_{q}^{1}(Z) \\
=\left\{\sum_{p=0}^{q} \sum_{1 \leqslant k_{1}<\cdots<k_{p} \leqslant q} \rho_{k_{1}}^{a} * \cdots * \rho_{k_{p}}^{a} * \prod_{i \neq k_{1}, \ldots, k_{p}} *\left(\delta_{0}+\rho_{i}^{s}\right)\right\} * \mu_{q}^{1}(Z) .
\end{aligned}
$$

Here $\Pi *$ is a convolution product. Let us denote the right-hand side of the above equalities by $(\Sigma)$. A term such that $\sum_{i=1}^{p} H_{k_{i}}=W$ disappears since $\rho_{k_{1}}^{a} * \rho_{k_{2}}^{a} * \cdots * \rho_{k_{p}}^{a} \ll \Lambda_{W}$ by Lemma 2.1. If $\operatorname{dim}\left(H:=\sum_{i=1}^{p} H_{k_{i}}\right) \leqslant m-1$ for $p \geqslant m-\ell$, then there are integers $\left\{j_{1}, \ldots, j_{m}\right\}$ in $\left\{k_{1}, \ldots, k_{p}\right\}$ such that $H=\sum_{i=1}^{m} H_{j_{i}}$. Let $\left\{i^{\prime}\right\}$ be a subsequence in $\{1, \ldots, q\}$ such that $H_{i^{\prime}} \subset H$. Sum of the terms in the linear subspace $H$ is not greater than

$$
b_{j_{1}} \cdots b_{j_{m}} \sum b_{k_{1}} \cdots b_{k_{p}}=b_{j_{1}} \cdots b_{j_{m}} \prod_{i}\left(1+b_{i}\right),
$$

where the sum is over $\left\{k_{1}, \ldots, k_{p}\right\} \subset\left\{i^{\prime}\right\} \backslash\left\{j_{1}, \ldots, j_{m}\right\}$ for $0 \leqslant p \leqslant q-m$ and the product is taken over $i \in\left\{i^{\prime}\right\} \backslash\left\{j_{1}, \ldots, j_{m}\right\}$. Since $b_{i} \leqslant\left(c_{i}\right)^{r} e^{c_{i}}$ and 
$D_{m-1}<\infty, \sum_{i^{\prime}} b_{i^{\prime}}<D_{m-1} e^{\left(D_{m-1}\right)^{1 / r}}=: D<\infty$. Hence we have

$$
(\Sigma) \leqslant(D+1)\left\{\sum_{p=0}^{m} \sum_{1 \leqslant j_{1}<\cdots<j_{p} \leqslant q} \prod_{i=1}^{p} \frac{b_{j_{i}}}{1+a_{j_{i}}}\right\} \prod_{i=1}^{q}\left(1+a_{i}\right) .
$$

We can choose a sequence of $m$-dimensional linear subspaces $\left\{W_{n}\right\}$ so that $\sum_{k} \nu_{k}\left(H_{k} \cap W_{n}\right)^{r} \rightarrow \infty$ as $n \rightarrow \infty$. Define $\mu_{1}^{(n)}, \mu_{2}^{(n)}, a_{i}^{(n)}, b_{i}^{(n)}$, and $c_{i}^{(n)}$ as above for $W_{n}$. Since $a_{i}^{(n)} \leqslant e^{c_{i}^{(n)}}, b_{i}^{(n)} \leqslant\left(c_{i}^{(n)}\right)^{r} e^{c_{i}^{(n)}}, c_{i}^{(n)} \leqslant\left(D_{m-1}\right)^{1 / r}$ and $\sum_{i=1}^{\infty}\left(c_{i}^{(n)}\right)^{r} \rightarrow \infty$ as $n \rightarrow \infty$ by the definition of $D_{m}$,

$$
(D+1) e^{-\sum_{k=1}^{\infty} c_{k}^{(n)}}\left\{\sum_{p=0}^{m} \sum_{1 \leqslant j_{1}<\cdots<j_{p}} \prod_{k=1}^{p} \frac{b_{j_{k}}^{(n)}}{1+a_{j_{k}}^{(n)}}\right\} \prod_{k=1}^{\infty}\left(1+a_{k}^{(n)}\right),
$$

upper bound of the mass of the singular part of $\mu_{1}^{(n)}$, tends to 0 as $n \rightarrow \infty$ by Lemma 2.5 . We have, by Lemma 2.2 ,

$$
\mu(Z)=\mu_{1}^{(n)} * \mu_{2}^{(n)}(Z) \rightarrow 0
$$

as $n \rightarrow \infty$.

Step 2. Let $\ell$ be an integer with $1 \leqslant \ell \leqslant d$. If $\sum_{k}\left(\nu_{\ell, k}\left(\mathbf{R}^{d}\right)\right)^{r}<\infty$, then set $W_{\ell}=\{0\}$. Assume that $\sum_{k}\left(\nu_{\ell, k}\left(\mathbf{R}^{d}\right)\right)^{r}=\infty$. If there is a $(d-1)$ dimensional linear subspace $K$ with $\sum_{k}\left(\nu_{\ell, k}\left(K^{c}\right)\right)^{r}<\infty$, then set $W=K$. Otherwise set $W_{\ell}=\mathbf{R}^{d}$. For the above $W, \sum_{k}\left(\nu_{\ell, k}(W)\right)^{r}=\infty$ holds by Lemma 2.3 and $\sum_{k}\left(\nu_{\ell, k}\left(W^{c}\right)\right)^{r}<\infty$ holds by the definition of $W$. If there is no $(d-2)$-dimensional linear subspace $K$ with $\sum_{k}\left(\nu_{\ell, k}\left(W \cap K^{c}\right)\right)^{r}<\infty$, then set $W_{\ell}=W$. If there is a $(d-2)$-dimensional linear subspace $K$ such that $\sum_{k}\left(\nu_{\ell, k}\left(W \cap K^{c}\right)\right)^{r}<\infty$. Then by Lemma $2.3, \sum_{k}\left(\nu_{\ell, k}(W \cap K)\right)^{r}=\infty$. Set $W=K$. Repeating this procedure, we have the following two cases.

1. There is a linear subspace $W$ such that $\ell+1 \leqslant \operatorname{dim} W=m \leqslant d$, $\sum_{k}\left(\nu_{\ell, k}\left(W^{c}\right)\right)^{r}<\infty$ and $\sum_{k}\left(\nu_{\ell, k}\left(W \cap K^{c}\right)\right)^{r}=\infty$ for any $(m-1)$-dimensional linear subspace $K$ of $W$. Then set $W_{\ell}$ as such $W$ with largest dimension.

2. There is no such $W$ for $m \leqslant \ell+1$. In this case, there is an $H_{\ell, k}$ such that $\nu_{\ell, k}\left(H_{\ell, k}\right)=\infty$ and $\sum_{j \neq k} \nu_{\ell, j}\left(H_{\ell, j}\right)^{r}<\infty$. Set $W_{\ell}=H_{\ell, k}$. If $W_{\ell}=\mathbf{R}^{d}$, then $\mu \ll \Lambda_{\mathbf{R}^{d}}$ by Step 1. If $\operatorname{dim} W_{\ell}<d$, then by the induction hypothesis, $\mu_{\ell}$ corresponding to $\sum_{k} \nu_{\ell, k}$ is absolutely continuous on $W_{\ell}$. Suppose that $\operatorname{dim}\left(\sum_{k=1}^{d} W_{k}\right)<d$. Then for $(d-1)$-dimensional linear subspace $K$ containing $\sum_{k=1}^{d} W_{k}$,

$$
\sum_{\ell=1}^{d} \sum_{k}\left(\nu_{\ell, k}\left(K^{c}\right)\right)^{r} \leqslant \sum_{\ell=1}^{d} \sum_{k}\left(\nu_{\ell, k}\left(\left(W_{\ell}\right)^{c}\right)\right)^{r}<\infty .
$$

This contradicts the assumption 2. Therefore, $\operatorname{dim}\left(\sum_{k=1}^{d} W_{k}\right)=d$ and $\mu \ll$ $\Lambda_{\mathbf{R}^{d}}$ by Lemma 2.1. Theorem 1.1 is proved. 
$\mathrm{Pr}$ o of of $\mathrm{Th}$ e or e $\mathrm{m}$ 1.2. We prove the theorem also by induction in $d$. If $d=1$, then the conclusion is obviously valid. Assume that the theorem is valid for dimension less than $d \geqslant 2$.

Step 1 . We prove the following statement: Let $1 \leqslant \ell \leqslant d-1$. If

$$
\nu^{(\ell)}\left(K^{c}\right)=\infty
$$

for any $(d-1)$-dimensional linear subspace $K$ in $\mathbf{R}^{d}$, then $\mu \ll \Lambda_{\mathbf{R}^{d}}$.

We denote $H_{k}^{(\ell)}$ by $H_{k}$ and $\nu^{(\ell)}$ by $\nu$. Let

$D_{m}:=\sup \left\{\nu(W): W\right.$ is an $m$-dimensional linear subspace of $\left.\mathbf{R}^{d}\right\}$.

Note that $\nu(W)=\sum_{H_{k} \subset W} \nu\left(H_{k} \cap W\right)$. We divide into the following cases:

$$
D_{1} \leqslant \cdots \leqslant D_{m-1}<\infty=D_{m}=\cdots=D_{d}, \quad m=\ell, \ell+1, \ldots, d .
$$

1. Assume the above case. Fix an $m$-dimensional linear subspace $W$ of $\mathbf{R}^{d}$ satisfying $\nu(W)>0$. Let $T=T_{W^{\perp}}$. Let $\sigma_{1}=\left.\nu\right|_{W}$ and $\sigma_{2}=\left.\nu\right|_{W^{c}}$ and let $\mu_{1}$ and $\mu_{2}$ be infinitely divisible distributions determined through (1) by $\sigma_{1}$ and $\sigma_{2}$, respectively. Note that $\operatorname{dim} W^{\perp}=d-m \leqslant d-1$. By the assumption (5), $\left(T \sigma_{2}\right)\left(K^{c}\right)=\nu^{(\ell)}\left(W^{c} \cap\left(T^{-1}(K)\right)^{c}\right)=\infty$ for any $(d-m-1)$ dimensional linear subspace $K$ of $W^{\perp}$ since $\operatorname{dim} T^{-1}(K)=d-1$ and $W^{c} \supset$ $\left(T^{-1}(K)\right)^{c}$. Let $\left\{V_{k}\right\}$ be a sequence of all $j$-dimensional linear subspaces of $W^{\perp}$ such that $0<\left(T \sigma_{2}\right)^{(j)}\left(V_{k}\right) \leqslant \infty$. Note that $V_{k}=T\left(H_{p}^{(\ell)}\right)$ for at least one $p$. Suppose that $V$ be an $r$-dimensional $(1 \leqslant j \leqslant r<d-m)$ linear subspace of $W^{\perp}$ such that $\left(T \sigma_{2}\right)^{(j)}(V)>0$. Let $S$ be an orthogonal projection of $W^{\perp}$ to $V^{\perp}$. Let $\left\{U_{q}\right\}$ be the sequence of all $i$-dimensional linear subspaces of $V^{\perp}$ such that $\left(S\left(\left.\left(T \sigma_{2}\right)^{(j)}\right|_{V^{c} \cap W^{\perp}}\right)\right)^{(i)}\left(U_{q}\right)>0$. Note that $U_{q}=(S T)\left(H_{p}\right)$ for at least one $p$ and $\left\{U_{q}\right\}$ is a subsequence of $\left\{S T\left(H_{p}\right)\right\}$. We have

$$
\begin{aligned}
\left(S\left(\left.\left(T \sigma_{2}\right)^{(j)}\right|_{V^{c} \cap W^{\perp}}\right)\right)^{(i)}(\cdot) & =\left(T \sigma_{2}\right)^{(j)}\left(S^{-1}(\cdot) \cap\left(\bigcup_{k^{\prime}} V_{k^{\prime}}\right) \cap V^{c} \cap W^{\perp}\right) \\
& =\sigma_{2}\left(T^{-1}\left(S^{-1}(\cdot) \cap\left(\bigcup_{k^{\prime}} V_{k^{\prime}}\right) \cap V^{c} \cap W^{\perp}\right)\right. \\
& =\nu\left((S T)^{-1}(\cdot) \cap\left(\bigcup_{k^{\prime}} T^{-1}\left(V_{k^{\prime}}\right)\right) \cap(V \oplus W)^{c}\right) \\
& =\left((S T)\left(\left.\nu\right|_{(V \oplus W)^{c}}\right)\right)^{(i)}(\cdot) .
\end{aligned}
$$

Here, $\left\{V_{k^{\prime}}\right\}$ is a subsequence of $\left\{V_{k}\right\}$ such that $\operatorname{dim} S\left(V_{k^{\prime}}\right)=i$ and $\left\{T^{-1}\left(V_{k^{\prime}}\right)\right\}$ is a subsequence of $\left\{H_{k}\right\}$. Hence $T \sigma_{2}$ satisfies the assumption by the assumption for the original $\nu$ regarding $S T$ as $T$. By the induction hypothesis, $T \mu \ll \Lambda_{W^{\perp}}$. 
2. Now, we consider $\mu_{1}$. Relabel $H_{k}$ in $W$ as $\left\{H_{k}\right\}$ and let $\nu_{1, k}=\left.\sigma_{1}\right|_{H_{k}}$. Let $c_{k}:=\nu_{1, k}\left(H_{k}\right), \rho_{k}=\sum_{j=1}^{\infty} \nu_{1, k}^{j *} / j$ !. Let $b_{k}=\rho_{k}\left(H_{k}\right)=e^{c_{k}}-1$. Define $\bar{\mu}_{q}$ by

$$
\mu_{1}=e^{-\sum_{k=1}^{q} c_{k}}\left(\delta_{0}+\rho_{1}\right) *\left(\delta_{0}+\rho_{2}\right) * \cdots *\left(\delta_{0}+\rho_{q}\right) * \bar{\mu}_{q} .
$$

Let $Z$ be a Borel set of $W$ with $\Lambda_{W}(Z)=0$. We have

$$
\begin{aligned}
& \left(\delta_{0}+\rho_{1}\right) *\left(\delta_{0}+\rho_{2}\right) * \cdots *\left(\delta_{0}+\rho_{q}\right) * \bar{\mu}_{q}(Z) \\
& \quad=\left\{\delta_{0}+\sum_{p=1}^{q} \sum_{1 \leqslant j_{1}<\cdots<j_{p} \leqslant q} \rho_{j_{1}} * \cdots * \rho_{j_{p}}\right\} * \bar{\mu}_{q}(Z) .
\end{aligned}
$$

For the term with $\operatorname{dim}\left(\sum_{k=1}^{p} H_{j_{k}}\right) \leqslant m-1(p \leqslant m)$, there is a subset $\left\{j_{k_{1}}, \ldots, j_{k_{m}}\right\}$ of $\left\{j_{1}, \ldots, j_{p}\right\}$ such that $\sum_{k=1}^{p} H_{j_{k}}=\sum_{i=1}^{m} H_{j_{k_{i}}}$. The sum of such terms with the same $\left\{j_{k_{1}}, \ldots, j_{k_{m}}\right\}$ is not greater than

$$
\begin{aligned}
& \left(e^{c_{j_{k_{1}}}}-1\right) \cdots\left(e^{c_{j_{k_{m}}}}-1\right) \sum_{p=0}^{q-m} \sum \rho_{j_{1}}\left(H_{j_{1}}\right) \cdots \rho_{j_{p}}\left(H_{j_{p}}\right) \\
& \leqslant b_{j_{k_{1}}} \cdots b_{j_{k_{m}}} \prod_{k^{\prime}}\left(\delta_{0}+\rho_{k^{\prime}}\right)\left(H_{k}\right) \leqslant b_{j_{k_{1}}} \cdots b_{j_{k_{m}}} e^{D_{m-1}} .
\end{aligned}
$$

Here, the sum $\sum_{p=0}^{q-m} \sum$ is taken for $j_{1}, \ldots, j_{p}$ such that $\sum_{i=1}^{p} H_{j_{i}} \subset \sum_{i=1}^{m} H_{j_{k_{i}}}$ and the product $\prod_{k^{\prime}}$ is taken for all $k^{\prime}$ such that $H_{k^{\prime}} \subset \sum_{i=1}^{m} H_{j_{k_{i}}}$. Hence the sum of terms $\rho_{j_{1}} * \cdots * \rho_{j_{p}} * \bar{\mu}_{q}(Z)$ with $\operatorname{dim}\left(\sum_{i=1}^{p} H_{j_{i}}\right)<m$ is bounded by

$$
\sum_{p=1}^{m} \sum_{1 \leqslant j_{1}<\cdots<j_{p} \leqslant q} b_{j_{1}} \cdots b_{j_{p}} e^{D_{m-1}}
$$

Next we consider a term with $\operatorname{dim}\left(\sum_{i=1}^{p} H_{j_{i}}\right)=m$. Let $L\left[x_{1}, \ldots, x_{p}\right]$ be the linear subspace spanned by $x_{1}, \ldots, x_{p}$. Let

$$
K_{p, r}=\left\{\left(x_{1}, \ldots, x_{p}\right) \in \prod_{i=1}^{p} H_{j_{i}}: \operatorname{dim} L\left[x_{1}, \ldots, x_{p}\right]=r\right\}
$$

and $K_{p}\left(i_{1}, \ldots, i_{r}\right)=\left\{\left(x_{1}, \ldots, x_{p}\right) \in K_{p, r}: x_{i_{1}}, \ldots, x_{i_{r}}\right.$ are linearly independent $\}$. We have

$$
\begin{gathered}
\int_{K_{p}\left(i_{1}, \ldots, i_{r}\right)} \prod_{k=1}^{p} \rho_{k}\left(d x_{k}\right)=\int_{K_{p}\left(i_{1}, \ldots, i_{r}\right)} \prod_{k=1}^{r} \rho_{j_{i_{k}}}\left(d x_{i_{j}}\right) \prod_{k \neq i_{1}, \ldots, i_{r}} \rho_{j_{k}}\left(d x_{k}\right) \\
=\int_{K\left(H_{i_{1}}, \ldots, H_{i_{r}}\right)} \prod_{k=1}^{r} \rho_{j_{i_{k}}}\left(d x_{i_{k}}\right) \prod_{k \neq i_{1}, \ldots, i_{r}} \int_{L\left[x_{i_{1}}, \ldots, x_{i_{r}}\right]} \rho_{j_{k}}\left(d x_{k}\right) .
\end{gathered}
$$


Assume that $r<m$. If there is $k \neq i_{1}, \ldots, i_{r}$ such that $H_{k} \nsubseteq \subseteq L\left[x_{i_{1}}, \ldots, x_{i_{r}}\right]$, then $\prod_{k \neq i_{1}, \ldots, i_{r}} \int_{L\left[x_{i_{1}}, \ldots, x_{i_{r}}\right]} \rho_{k}\left(d x_{k}\right)=0$ and hence

$$
\int_{K_{p}\left(i_{1}, \ldots, i_{r}\right)} \prod_{k=1}^{p} \rho_{j_{k}}\left(d x_{k}\right) \leqslant \int_{K\left(H_{i_{1}}, \ldots, H_{i_{r}}\right)} \prod_{k=1}^{r} \rho_{j_{i_{k}}}\left(d x_{i_{k}}\right) e^{D_{m-1}} \leqslant \prod_{j=1}^{r} b_{i_{j}} e^{D_{m-1}} .
$$

Hence

$$
\int_{K_{p, r}} \prod_{k=1}^{p} \rho_{j_{k}}\left(d x_{k}\right) \leqslant e^{D_{m-1}} \sum_{1 \leqslant i_{1}<\cdots<i_{r} \leqslant p} \prod_{j=1}^{r} b_{i_{j}} .
$$

Let $r=m$. Then, we have

$$
\begin{aligned}
& \int_{K_{p}\left(i_{1}, \ldots, i_{m}\right)} 1_{Z}\left(\sum_{k=1}^{m} x_{i_{k}}+x+y\right) \prod_{k=1}^{m} \rho_{j_{i_{k}}}\left(d x_{i_{k}}\right)\left(\prod_{k} \rho_{j_{k}}\left(d x_{k}\right)\right) \bar{\mu}_{q}(d y) \\
& =\int_{K_{p}\left(i_{1}, \ldots, i_{m}\right)} 1_{Z-x-y}\left(\sum_{k=1}^{m} x_{i_{k}}\right) \prod_{k=1}^{m} \rho_{j_{i_{k}}}\left(d x_{i_{k}}\right)\left(\prod_{k} \rho_{j_{k}}\left(d x_{k}\right)\right) \bar{\mu}_{q}(d y)=0
\end{aligned}
$$

by the assumption with $W=\{0\}$ and $T=I$, where $I$ is an identity operator on $\mathbf{R}^{d}$ and $x=\sum_{k \in\{1, \ldots, p\} \backslash\left\{i_{1}, \ldots, i_{m}\right\}} x_{k}$. Letting $q \rightarrow \infty$, we have by (6)-(8),

$$
\mu_{1}(Z) \leqslant 2\left(\sum_{j=1}^{m}\left(\sum_{k=1}^{\infty} b_{k}\right)^{j} e^{D_{m-1}}\right) .
$$

As in Step 1 in the proof of Theorem 1.1, we have that $\mu(Z)=0$ for Borel set $Z$ with $\Lambda_{\mathbf{R}^{d}}(Z)=0$.

Step 2. Apply Step 2 in the proof of Theorem 1.1 setting $r=1$. We have $\mu \ll \Lambda_{\mathbf{R}^{d}}$. Theorem 1.2 is proved.

4. Absolute continuity under GPDACR. Let $S$ be a Borel measurable subset of $(d-1)$-dimensional unit sphere center at the origin. We call a map

$$
\varphi(u, \xi):(0, \infty) \times S \rightarrow \mathbf{R}^{d} \backslash\{0\}
$$

generalized polar coordinate on $\mathbf{R}^{d}$ of class $C^{1}$ with $S$ if it satisfies the following three conditions:

1) $\varphi$ is measurable with respect to the $\xi$, class $C^{1}$ with respect to the $u$, one to one, $\varphi(0+, \xi)=0, \varphi(1, \xi)=\xi$, and $\partial \varphi(u, \xi) / \partial u \neq 0$.

2) For $\xi \in S$ and a subset $A$ of $\mathbf{R}^{d}$, set

$$
C_{\varphi}(\xi, A)=\left\{u \in(0, \infty): \frac{\partial}{\partial u} \varphi(u, \xi) \in A\right\}
$$

For every linear subspace $H$ and any $\xi \in S$, the set $C_{\varphi}(\xi, H)$ coincides with $(0, \infty)$ or is of Lebesgue measure 0 . 
3) For any $\xi_{1}, \ldots, \xi_{k} \in S(2 \leqslant k \leqslant d)$, let $F_{k}\left(\xi_{1}, \ldots, \xi_{k}\right)$ be a subset of $(0, \infty)^{k}$ consisting of $\left(u_{1}, \ldots, u_{k}\right)$ such that $\left\{\varphi\left(u_{1}, \xi_{1}\right), \ldots, \varphi\left(u_{k}, \xi_{k}\right)\right\}$ are linearly independent and $\left\{\partial \varphi\left(u_{1}, \xi_{1}\right) / \partial u_{1}, \ldots, \partial \varphi\left(u_{k}, \xi_{k}\right) / \partial u_{k}\right\}$ are linearly dependent. Then the $k$-dimensional Lebesgue measure of the set $F_{k}\left(\xi_{1}, \ldots, \xi_{k}\right)$ is zero.

Ex a m p l e $4.1[2]$. Let $Q$ be a $d \times d$ matrix whose all eigenvalues have positive real parts. Then $\varphi(u, \xi)=u^{Q} \xi:=e^{(\ln u) Q} \xi$ is a generalized polar coordinate on $\mathbf{R}^{d}$ with $S=S_{Q}=\left\{\xi \in \mathbf{R}^{d}:|\xi|=1,\left|u^{Q} \xi\right|>\right.$ 1 for every $u>1\}$.

We say that a Lévy measure $\nu$ has a generalized polar decomposition with absolutely continuous radial part (GPDACR) if there is a generalized polar coordinate $(S, \varphi)$ of class $C^{1}$ such that for $B \in \mathscr{B}\left(\mathbf{R}^{d}\right)$,

$$
\nu(B)=\int_{S} \lambda(d \xi) \int_{(0, \infty)} 1_{B}(\varphi(u, \xi)) \ell(\xi, u) d u
$$

where $\lambda(d \xi)$ is a probability measure on $S$ and $\ell(\xi, u)$ is nonnegative and measurable in each variable satisfying $\int_{(0, \infty)} \widetilde{\ell}(\xi, u) d u<\infty$ for $\lambda$-a.a. $\xi$, where

$$
\widetilde{\ell}(\xi, u)=|\varphi(u, \xi)|^{2}\left(1+|\varphi(u, \xi)|^{2}\right)^{-1} \ell(\xi, u) .
$$

In [10], the following fact is proved.

Assume that $\nu\left(\mathbf{R}^{d}\right)=\infty$ and GPDACR for $\nu$. Then the following holds.

1. If $\nu$ is not concentrated on a proper linear subspace of $\mathbf{R}^{d}$ and either $\nu(H)=0$ or $\nu(H)=\infty$ for every linear subspace $H$, then $\mu \ll \Lambda_{\mathbf{R}^{d}}$.

2. If $\nu(H)=0$ for every $(d-2)$-dimensional linear subspace $H$ of $\mathbf{R}^{d}$ and there is no $(d-1)$-dimensional linear subspace $H$ such that $\nu\left(\mathbf{R}^{d} \backslash H\right)<\infty$, then $\mu \ll \Lambda_{\mathbf{R}^{d}}$.

We extend the above results as the following Theorems 4.1 and 4.2.

Theorem 4.1. Assume GPDACR for $\nu$ and

$$
\sum_{\ell=1}^{d-1} \sum_{k}\left(\nu^{(\ell)}\left(H_{k}^{(\ell)} \cap K^{c}\right)\right)^{d-1}+\nu^{(d)}\left(\mathbf{R}^{d}\right)=\infty
$$

for any $(d-1)$-dimensional linear subspace $K$ of $\mathbf{R}^{d}$. Then, $\mu \ll \Lambda_{\mathbf{R}^{d}}$.

$\mathrm{P}$ r o o f. If $\nu$ satisfies GPDACR condition, then for $1 \leqslant \ell \leqslant d-1$, $\left(\left.\widetilde{\nu}^{(\ell)}\right|_{H_{k}^{(\ell)}}\right)^{\ell *} \ll \Lambda_{H_{k}^{(\ell)}}$ for each $k$ by the proof of Theorem 1 in [9]. Then $\left(\left.\widetilde{\nu}^{(\ell)}\right|_{H_{k}^{(\ell)}}\right)^{(d-1) *} \ll \Lambda_{H_{k}^{(\ell)}}$ also holds for each $k$. Under the condition (3), the assumption 2 of Theorem 1.1 with $r=d-1$ holds and we get the conclusion. Theorem 4.1 is proved.

Theorem 4.2. Assume GPDACR for $\nu$. Moreover, assume that for any linear subspace $H$ satisfying $0<\nu\left(H \backslash \bigcup_{k} H_{k}\right) \leqslant \infty$, where $\left\{H_{k}\right\}$ is the 
totality of proper linear subspaces of $H, T \varphi(\cdot, \cdot)$ with $T=T_{H^{\perp}}$ satisfies the condition 3 of generalized polar coordinate. Then $\mu \ll \Lambda_{\mathbf{R}^{d}}$ if and only if

$$
\nu\left(K^{c}\right)=\infty
$$

for any $(d-1)$-dimensional linear subspace $K$ of $\mathbf{R}^{d}$.

$\mathrm{P}$ r o o f. We show that the assumption in Theorem 1.2 is satisfied. Let $W=\{0\}$. Then $W^{\perp}=\mathbf{R}^{d}$. Let $V_{j}=H_{j}^{(\ell)}$ and $V:=\sum_{j=1}^{r} V_{i_{j}}$ with $\operatorname{dim} V=r$. Let $\left[\mathbf{h}_{1}, \ldots, \mathbf{h}_{r}\right]$ be an orthonormal basis of $V$. Since $\sum_{i=1}^{r} \varphi\left(u_{i}, \xi_{i}\right) \in V$ for $\xi_{1}, \ldots, \xi_{r} \in K\left(i_{1}, \ldots, i_{r}\right)$, we can write

$$
\sum_{i=1}^{r} \varphi\left(u_{i}, \xi_{i}\right)=\sum_{j=1}^{r} c_{j}(\mathbf{u}) \mathbf{h}_{j},
$$

where $\mathbf{u}=\left(u_{1}, \ldots, u_{r}\right)$. Define a linear map $h: \mathbf{R}^{r} \rightarrow V$ by $h(\mathbf{v})=\sum_{j=1}^{r} v_{j} \mathbf{h}_{j}$ for $\mathbf{v}=\left(v_{1}, \ldots, v_{r}\right)$, which is an isomorphism. Let $\mathbf{c}(\mathbf{u})=\left(c_{1}(\mathbf{u}), \ldots, c_{r}(\mathbf{u})\right)$. Then $\mathbf{c}$ is continuously differentiable. Let $E=E_{r}\left(\xi_{1}, \ldots, \xi_{r}\right)=(0, \infty)^{r} \backslash F_{r}$, where $F_{r}=F_{r}\left(\xi_{1}, \ldots, \xi_{r}\right)$ is the set defined in property 3 of generalized polar coordinate $\varphi$. Let $a_{i j}(\mathbf{u})=\frac{\partial}{\partial u_{i}} c_{j}(\mathbf{u})$. Then the matrix $A(\mathbf{u})=\left(a_{i j}(\mathbf{u})\right)$ is invertible if $\mathbf{u} \in E$. Then, we have

$$
\begin{aligned}
\int_{(0, \infty)^{r}} 1_{Z}\left(\sum_{i=1}^{r} \varphi\left(u_{i}, \xi_{i}\right)\right) \prod_{i=1}^{r} d u_{i} & =\int_{E} 1_{Z}(h(\mathbf{c}(\mathbf{u}))) d \mathbf{u} \\
& =\int_{\mathbf{c}(E)} 1_{h^{-1}(Z)}(\mathbf{v})\left|\operatorname{det}\left(A^{-1}(\mathbf{v})\right)\right| d \mathbf{v}=0 .
\end{aligned}
$$

Since

$$
\begin{aligned}
& \int_{K\left(V_{i_{1}}, \ldots, V_{i_{r}}\right)} 1_{Z}\left(\sum_{j=1}^{r} x_{j}\right) \prod_{j=1}^{r} \widetilde{\nu}^{(\ell)}\left(d x_{j}\right) \\
& \leqslant \int_{\prod_{j=1}^{r} V_{i_{j}}} \prod_{j=1}^{r} \lambda^{(\ell)}\left(d \xi_{j}\right) \int_{E} 1_{Z}\left(\sum_{j=1}^{r} \varphi\left(u_{j}, \xi_{j}\right)\right) \prod_{j=1}^{r} \tilde{\ell}\left(u_{j}, \xi_{j}\right) d u_{j},
\end{aligned}
$$

we have

$$
\int_{K\left(V_{i_{1}}, \ldots, V_{i_{r}}\right)} 1_{Z}\left(\sum_{j=1}^{r} x_{j}\right) \prod_{j=1}^{r} \widetilde{\nu}^{(\ell)}\left(d x_{j}\right)=0 .
$$

Here, $\tilde{\ell}$ is defined by (9).

Let $W$ be an $m$-dimensional linear subspace, where $\ell \leqslant m \leqslant d$. Let $\left\{V_{k}\right\}$ be a sequence of all $j$-dimensional linear subspaces of $W^{\perp}$ such that $0<$ $T \nu_{\ell}\left(V_{k}\right) \leqslant \infty$, where $T \nu_{\ell}$ is defined in Theorem 1.2. Suppose that $\operatorname{dim}(V:=$ $\left.\sum_{i=1}^{r} V_{k_{i}}\right)=r$. Then in the above argument for $W=\{0\}, \Lambda_{\mathbf{R}^{r}}\left(F_{r}\right)=0$ by the assumption. Hence, the assumption of Theorem 1.2 is satisfied in this case. Theorem 4.2 is proved. 
In the above theorems, we do not assume the following divergence condition:

$$
\int_{(0, \infty)} \ell(\xi, u) d u=\infty \text { for } \lambda \text {-a.a. } \xi
$$

which is crucial in the proof of Theorem 27.10 in Sato's book [4].

5. An application to OU type processes. Let $Q$ be a $d \times d$-matrix and let $Z(t)$ be a Lévy process on $\mathbf{R}^{d}$ with (1). Let $X(t)$ be a stochastic process defined by a stochastic differential equation

$$
d X(t)=-Q X(t) d t+d Z(t), \quad X(0)=0 .
$$

Such a process is called a process of Ornstein-Uhlenbeck type in [5]. Let $\mu_{t}, t>0$, be the distribution of $X(t)$. It is known [5] that $\mu_{t}$ is infinitely divisible and its Lévy measure $\nu_{t}$ is represented as

$$
\nu_{t}(B)=\int_{\mathbf{R}^{d}} \nu(d y) \int_{0}^{t} 1_{B}\left(e^{-s Q} y\right) d s .
$$

If all eigenvalues of $Q$ have positive real parts, then $\varphi(u, \xi)=u^{Q} \xi$ is a generalized polar coordinate of class $C^{1}$ with $S=S_{Q}$ and by the above representation, we have

$$
\nu_{t}(B)=\int_{S_{Q}} \lambda(d \xi) \int_{0}^{\infty} 1_{B}\left(u^{Q} \xi\right) \frac{k_{\xi}(u)-k_{\xi}\left(e^{t} u\right)}{u} d u
$$

where $\lambda$ is a probability measure on $S_{Q}, k_{\xi}(u)$ is nonnegative, Borel measurable in $\xi$ and nonincreasing in $u$ and satisfies

$$
\int_{(0, \infty)} \frac{\left|u^{Q} \xi\right|^{2}}{1+\left|u^{Q} \xi\right|^{2}} \frac{k_{\xi}(u)-k_{\xi}\left(e^{t} u\right)}{u} d u<\infty
$$

Note that the divergence condition (11) is not satisfied in general in this case. Hence, the result on absolute continuity in [4] cannot be applied to this case.

Theorem 5.1. Assume that $Q$ is invertible. Then $\mu_{t} \ll \Lambda_{\mathbf{R}^{d}}$ if and only if $\nu_{t}\left(K^{c}\right)=\infty$ for every $(d-1)$-dimensional linear subspace $K$.

P r o o f. We show that the assumption in Theorem 1.2 is satisfied.

For the proof, we use an argument similar to the proof of Theorem 4.2. However, it is not clear whether GPDACR is satisfied in this situation. Therefore, we use the representation (12) of $\nu_{t}$ instead of the use of generalized polar coordinate decomposition.

1. Let $W=\{0\}$. That is, $W^{\perp}=\mathbf{R}^{d}$ and $T=I$. Let $V_{i}=H_{i}^{(\ell)}$ and $V:=\sum_{j=1}^{r} V_{i_{j}}$ with $\operatorname{dim} V=r \leqslant \ell$. For $H=H_{i}^{(\ell)}$, the totality $H_{0}$ of $y \in H$ such that $e^{t Q} y \in H$ for all $t>0$ is a linear subspace of $H$. If $H$ is not 
$Q$-invariant, then $H_{0}$ is a proper subspace of $H$. Then $\nu_{t}^{(\ell)}\left(H_{0}\right)=0$. If $y \in H \backslash H_{0}$, then $e^{t Q} y \notin H$ for a.a. $t>0$ and hence $\nu_{t}^{(\ell)}\left(H \backslash H_{0}\right)=0$ by (12). Hence $H$ is $Q$-invariant and thus $V$ is $Q$-invariant. Let $\left[\mathbf{h}_{1}, \ldots, \mathbf{h}_{r}\right]$ be an orthonormal basis of $V$. Since $\sum_{i=1}^{r} e^{-u_{i} Q} y_{i} \in V$ for $y_{1}, \ldots, y_{r} \in V$, we can represent as $\sum_{i=1}^{r} e^{-u_{i} Q} y_{i}=\sum_{j=1}^{r} c_{j}(\mathbf{u}) \mathbf{h}_{j}$, where $\mathbf{u}=\left(u_{1}, \ldots, u_{r}\right)$. Define a linear map $h: \mathbf{R}^{r} \rightarrow V$ by $h(\mathbf{v})=\sum_{j=1}^{r} v_{j} \mathbf{h}_{j}$ for $\mathbf{v}=\left(v_{1}, \ldots, v_{r}\right)$, which is an isomorphism. Let $\mathbf{c}(\mathbf{u})=\left(c_{1}(\mathbf{u}), \ldots, c_{r}(\mathbf{u})\right)$. Then $\sum_{i=1}^{r} e^{-u_{i} Q} y_{i}=$ $h(\mathbf{c}(\mathbf{u}))$ and $\mathbf{c}$ is continuously differentiable. Let $a_{i j}(\mathbf{u})=\frac{\partial}{\partial u_{i}} c_{j}(\mathbf{u})$. If $e^{-u_{1} Q} y_{1}, \ldots, e^{-u_{r} Q} y_{r}$ are linearly independent, then since $Q$ is invertible, $Q e^{-u_{1} Q} y_{1}, \ldots, Q e^{-u_{r} Q} y_{r}$ are linearly independent and the matrix $A(\mathbf{u})=$ $\left(a_{i j}(\mathbf{u})\right)$ is invertible. Let $F\left(y_{1}, \ldots, y_{r}\right)$ be the set of $\left(u_{1}, \ldots, u_{r}\right) \in[0, t]^{r}$ such that $e^{-u_{1} Q} y_{1}, \ldots, e^{-u_{r} Q} y_{r}$ are linearly independent. Then, $F\left(y_{1}, \ldots, y_{r}\right)$ is empty or an open set and we have

$$
\begin{aligned}
& \int_{F\left(y_{1}, \ldots, y_{r}\right)} 1_{Z}\left(\sum_{i=1}^{r} e^{-u_{i} Q} y_{i}\right) \prod_{i=1}^{r} d u_{i} \\
& =\int_{\mathbf{c}\left(F\left(y_{1}, \ldots, y_{r}\right)\right)} 1_{h^{-1}(Z)}(\mathbf{v})\left|\operatorname{det}\left(A^{-1}(\mathbf{v})\right)\right| d \mathbf{v}=0
\end{aligned}
$$

for Borel set $Z$ in $V$ with $\Lambda_{V}(Z)=0$. Here, $\mathbf{v}=\mathbf{c}(\mathbf{u})$. Since

$$
\begin{aligned}
& \int_{K\left(V_{i_{1}}, \ldots, V_{i_{r}}\right)} 1_{Z}\left(\sum_{j=1}^{r} x_{j}\right) \prod_{j=1}^{r} \widetilde{\nu}_{t}^{(\ell)}\left(d x_{j}\right) \\
& \leqslant \int_{\prod_{j=1}^{r} V_{i_{j}}} \prod_{j=1}^{r} \widetilde{\nu}_{t}^{(\ell)}\left(d y_{j}\right) \int_{F\left(y_{1}, \ldots, y_{r}\right)} 1_{Z}\left(\sum_{j=1}^{r} e^{-u_{j} Q} y_{j}\right) \prod_{j=1}^{r} d u_{j},
\end{aligned}
$$

we have

$$
\int_{K\left(V_{i_{1}}, \ldots, V_{i_{r}}\right)} 1_{Z}\left(\sum_{j=1}^{r} x_{j}\right) \prod_{j=1}^{r} \widetilde{\nu}_{t}^{(\ell)}\left(d x_{j}\right)=0
$$

for Borel set $Z$ in $V$ with $\Lambda_{V}(Z)=0$.

2. Let $W$ be an $m$-dimensional linear subspace of $\mathbf{R}^{d}$ such that $\nu_{t}^{(\ell)}(W \cap$ $\left.\left(\bigcup_{k} H_{k}^{(\ell)}\right)\right)>0$ and let $T=T_{W^{\perp}}$. Then, since $W$ is $Q$ invariant and $T Q=$ $T Q T, T X_{t}$ satisfies $d\left(T X_{t}\right)=T Q\left(T X_{t}\right) d t+d\left(T Z_{t}\right)$ and $T X_{t}$ is $W^{\perp}$-valued. The Lévy measure of the distribution of $T X_{t}$ is $T \nu_{t}$ and $T \nu_{t}$ has the same property as property 1 . Hence, by Theorem 1.2 , we have that $\mu \ll \Lambda_{\mathbf{R}^{d}}$. Theorem 5.1 is proved.

Note that the condition $\nu_{t}\left(K^{c}\right)=\infty$ for all $t>0$ is equivalent to the condition $\nu_{t}\left(K^{c}\right)$ for some $t>0$.

Bodnarchuk and Kulik [1] show that if $\nu\left(K^{c}\right)=\infty$ for every $(d-1)$ dimensional linear subspace $K$ (they call it Yamazato condition), then $\mu_{t} \ll$ $\Lambda_{\mathbf{R}^{d}}$ for all $t>0$ (Theorem 3 in [1]). Our assumption is weaker and the proof is elementary. The following example shows this situation and therefore their condition is not a necessary condition for the absolute continuity. 
E $\mathrm{x}$ a $\mathrm{m} \mathrm{ple} \mathrm{5.1.} \mathrm{Let} d=2$ and let

$$
Q=\left(\begin{array}{ll}
\alpha & 0 \\
0 & \beta
\end{array}\right), \quad \alpha>\beta>0 .
$$

Assume that $\nu$ is supported on the line $\{(x, x): x \in \mathbf{R}\}$ and $\nu\left(\mathbf{R}^{2}\right)=\infty$. Then $\nu$ does not satisfy Yamazato condition but $\nu_{t}$ does for $t>0$.

Simon [6] obtains a result quite similar to Theorem 5.1. However, the necessary and sufficient condition for the absolute continuity is stated in a different way.

\section{REFERENCES}

1. Bodnarchuk S. V., Kulyk O.M. Conditions for the existence and smoothness of the distribution density of the Ornstein-Uhlenbeck process with Lévy noise. - Theory Probab. Math. Statist., 2008/2009, v. 79, p. 23-38.

2. Hudson W. N., Mason J.D. Operator-stable laws. - J. Multivariate Anal., 1981, v. 11 , № 3 , p. 434-447.

3. Sato K. Absolute continuity of multivariate distribuions of class L. - J. Multivariate Anal., 1982, v. 12, № 1, p. 89-94.

4. Sato K. Lévy Processes and Infinitely Divisible Distributions. Cambridge: Cambridge Univ. Press, 1999, 486 p.

5. Sato K., Yamazato M. Operator-self-decomposable distributions as limit distributions of processes of Ornstein-Uhlenbeck type. - Stochastic Process. Appl., 1984, v. 17, p. $73-100$.

6. Simon T. On the absolute continuity of multidimensional Ornstein-Uhlenbeck processes. - Probab. Theory Related Fields (to appear).

7. Tucker H. G. Absolute continuity of infinitely divisible distributions. - Pacific J. Math., 1962, v. 12, p. 1125-1129.

8. Yamazato $M$. Absolute continuity of operator-self-decomposable distributions on $\mathbf{R}^{d}$. - J. Multivariate Anal., 1983, v. 13, № 4, p. 550-560.

9. Yamazato $M$. A simple proof of absolute continuity of multivariate operator-selfdecomposable distributions. - Bull. Nagoya Inst. Tech., 1992, № 44, p. 117-120.

10. Yamazato M. Absolute continuity of transition probabilities of multidimensional processes with stationary independent increments. - Теория вероятн. и ее примен., 1994, т. 39 , в. 2 , с. $347-354$. 